\title{
Announcement
}

\section{European Neuroendocrine Association (ENEA)}

The ENEA has been established and has held its First Symposium in Basel, March 4-7, 1984. The Second Symposium of the ENEA is scheduled to be held in Milan, fall 1985.

The Meeting will focus especially on hypothalamic regulatory peptides (chemistry, biochemistry, pharmacology, and clinical applications), but room will be also given to free communication sessions and/or symposia on topics of high interest in neuroendocrine research.

For further information on the Symposium write to: Dr. Euge-nio E. Müller, Department of Pharmacology, School of Medicine, University of Milan, via Vanvitelli 32,1-20129 Milan (Italy).

For information concerning affiliation to ENEA, please contact: Dr. A. Gomez-Pan, Departamento de Endocrinología, CNEQ, Pabellón 8, Facultad de Medicina, Madrid 3 (Spain), or Dr. M.F. Scanlon, Welsh National School of Medicine, Health Park, Cardiff, Wales (UK). 